APS

physics

This is the accepted manuscript made available via CHORUS, the article has been published as:

\title{
Nanomechanical Energy Storage in Twisted Nanotube Ropes
}

David Teich, Zacharias G. Fthenakis, Gotthard Seifert, and David Tománek

Phys. Rev. Lett. 109, 255501 - Published 20 December 2012

DOI: 10.1103/PhysRevLett.109.255501 


\title{
Deformation energetics of carbon nanotube ropes
}

\author{
David Teich, ${ }^{1}$ Zacharias G. Fthenakis, ${ }^{2}$ Gotthard Seifert, ${ }^{1}$ and David Tománek ${ }^{2, *}$ \\ ${ }^{1}$ Physikalische Chemie, Technische Universität Dresden, D-01062 Dresden, Germany \\ ${ }^{2}$ Physics and Astronomy Department, Michigan State University, East Lansing, Michigan 48824-2320, USA
}

\begin{abstract}
We determine the deformation energetics and energy density of twisted carbon nanotubes and nanotube ropes that effectively constitute a torsional spring. Using $a b$ initio and parameterized density functional calculations, we identify structural changes in these systems and determine their elastic limits. The deformation energy of twisted nanotube ropes contains contributions associated not only with twisting, but also with stretching, bending and compression of individual nanotubes. We quantify these energy contributions and show that their relative role changes with the number of nanotubes in the rope.
\end{abstract}

PACS numbers: 61.48.De, 62.25.-g, 61.46.-w

Graphitic carbon, including graphene and nanotubes, displays an unprecedented resilience in response to inplane deformations[1]. Numerous experimental studies have explored the response of nanotube ropes to axial stress $[2-5]$ and torsion[6, 7]. Theoretical studies have explored the response of nanotubes to deformations including stretching, twisting and bending [5, 8-15], and of infinite nanotube arrays to compression[16]. These calculations, mostly based on parameterized force fields and analytical models, have established the elastic constants and provided limited insight into the deformation behavior in the plastic regime. Nanotube bundles or ropes, which form by self-assembly of single-wall carbon nanotubes, should display a very different deformation behavior from isolated nanotubes when twisted. For one, twisting a rope results in a combination of stretching, twisting and bending of the constituent nanotubes[15]. Even more important is the interplay between different deformation modes in a rope, an important example being the tensile stress at the surface of a twisted rope that radially compresses the rope to minimize the energy. So far, little is known about the total deformation energy and how the relative role of the different deformation modes depends on the rope size. No information is available about the interplay between those deformation modes in ropes of different size and about the elastic limit and the microscopic decay mechanism of twisted nanotube ropes.

Here we use $a b$ initio and parameterized density functional calculations to determine the deformation energetics and energy density of twisted carbon nanotubes and nanotube ropes that effectively constitute a torsional spring. Our calculations not only identify the elastic range and deformation energy density of twisted carbon nanotubes and nanotube ropes, but also reveal their decay mechanism in the plastic regime. We demonstrate that the deformation energy of twisted nanotube ropes contains contributions associated not only with twisting, but also with stretching, bending and compression of individual nanotubes. Analysis of these energy contributions shows that their relative role changes with the num- ber of nanotubes in the rope.

Our total energy calculations for isolated and bundled carbon nanotubes are based on the $a b$ initio density functional theory (DFT), as implemented in the SIESTA code[17]. We used the Ceperley-Alder exchangecorrelation formalism[18] as parameterized by Perdew and Zunger[19] and norm-conserving Troullier-Martins pseudopotentials [20]. We utilized a double-zeta basis, including polarization orbitals, and periodic boundary conditions for all calculations. The Brillouin zone of the periodic nanotube array was sampled by a $2 \times 2 \times 8 k$-point grid. We limited the range of the localized orbitals in such a way that the energy shift caused by their spatial confinement was no more than $10 \mathrm{meV}[21]$. The charge density and potentials were determined on a real-space grid with a mesh cutoff energy of $100 \mathrm{Ry}$, which was sufficient to achieve a total energy convergence of better than $0.1 \mathrm{meV} /$ unit cell during the self-consistency iterations. All geometries have been optimized using the conjugate gradient method, until none of the residual HellmannFeynman forces exceeded $0.04 \mathrm{eV} / \AA$.

Since description of twisted nanotubes using conventional periodic boundary conditions requires very large unit cells in the axial direction, we studied deformations caused by torsion using the DFTB[22] adaptation of a simplified density functional theory with a local orbital basis to systems with helical symmetry[23-25]. This total energy functional had been applied successfully to a variety of carbon structures[22] and subsequently extended to accommodate van der Waals interactions[26], including their proper description in graphitic systems[26]. Use of helical symmetry, which allowed proper description of chiral nanotubes in the past[14, 25, 27], turned out also essential to efficiently describe the geometry and energy changes in twisted nanotubes and nanotube ropes.

Whereas past theoretical studies have mostly investigated deformations and the deformation energy associated with stretching and torsion of individual nanotubes[9-14], we focus here on nanotube arrays and ropes. Additional deformation energy is associated with the radial compression of a rope containing aligned nan- 

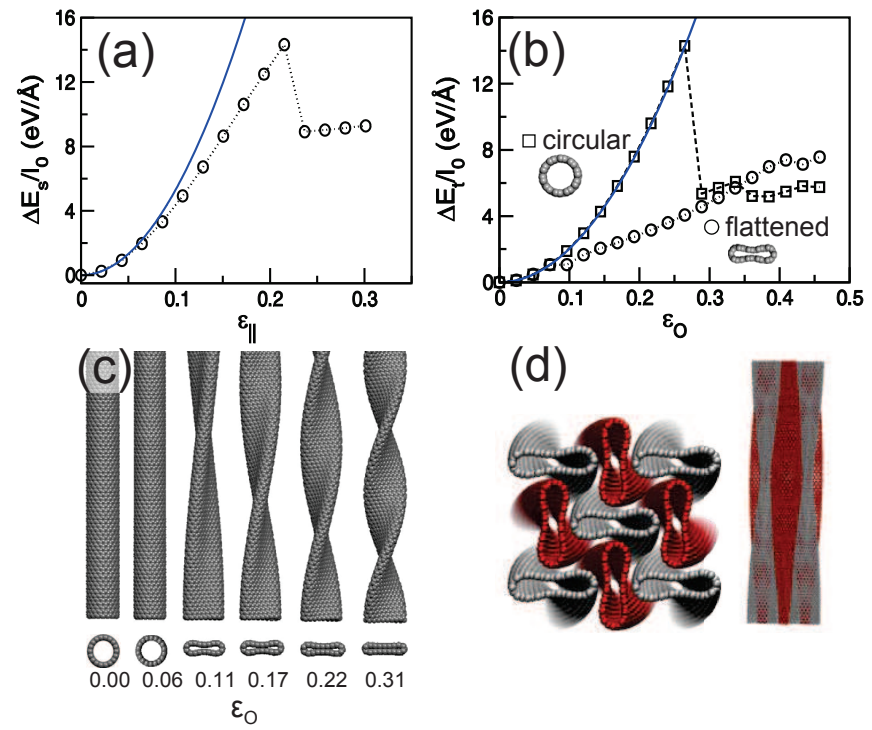

(d)

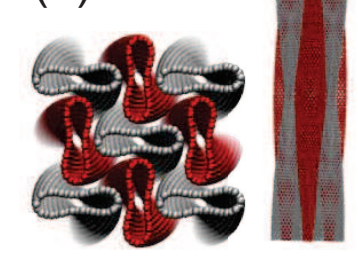

FIG. 1. (Color online) Deformation energy of an individual carbon nanotube subject to stretching and torsion. (a) Stretch energy per length $\Delta E_{s} / l_{0}$ of a $(10,10)$ nanotube as a function of axial strain $\epsilon_{\|}=l / l_{0}-1, l_{0}$ is the length of an unstrained nanotube segment. (b) Twist energy per length $\Delta E_{t} / l_{0}$ as a function of the dimensionless twist rate $\epsilon_{\circ}$ for an armchair $(10,10)$ nanotube. (c) Snap shots of a deforming $(18,0)$ nanotube at different twist rates in side and end-on view. (d) A possible space-filling compact arrangement of individually twisted $(18,0)$ carbon nanotubes in end-on and side view.

otubes. In the following we consider all these deformation modes in arrangements of the most abundant $(10,10)$ armchair nanotubes and their $(18,0)$ zigzag counterparts with the similar diameter of $1.4 \mathrm{~nm}$.

Numerical results for the stretch energy $\Delta E_{s}$ of a $(10,10)$ nanotube as a function of the axial strain $\epsilon_{\|}=$ $l / l_{0}-1$ are shown in Fig. 1(a). In our definition, $l$ is the actual and $l_{0}$ the initial length of an unstrained nanotube segment. Our calculation has been performed in supercell geometry with 4 primitive unit cells in the axial direction containing 160 atoms in total. Our results, displayed in Fig. 1(a), indicate an initial parabolic Hook's law behavior

$$
\Delta E_{s} / l_{0}=\alpha \epsilon_{\|}^{2}
$$

with $\alpha \approx 529.9 \mathrm{eV} / \AA$. This harmonic regime is followed by a softer response and eventual plastic deformation by dislocations[10] and fracture at strain values $\epsilon_{\|} \gtrsim \epsilon_{\|, \max }$. The estimated fracture strain value $\epsilon_{\|, \max } \approx 22 \%$ is unlikely to be achieved in reality, since finite temperature effects and presence of defects reduce it in the experiment to $\epsilon_{\|, \max } \approx 12 \%$ in the optimum case [2] or to even less. Even at the reduced value $\epsilon_{\|, \max }=12 \%$, stretching nanotubes to the elastic limit requires an energy investment of $3 \mathrm{MJ} / \mathrm{kg}$, which is typical of covalently bonded solids of light elements.
Bending a carbon nanotube is associated with the energy cost

$$
\Delta E_{b} / l_{0}=\beta\left(r_{0} / R\right)^{2},
$$

where $R$ is the local radius of curvature and $\beta \approx 256.6 \mathrm{eV} / \AA$. Since typical values of $R$ are significantly larger than the radius of a $(10,10)$ nanotube, $r_{0}=6.78 \AA$, the bending energy is usually quite small.

Much more important is the deformation energy $\Delta E_{t}$ associated with twisting a nanotube about its own axis. We first associate the angle $\varphi$ with the axial rotation of the nanotube cross-section along a tube segment of length $l_{0}$. For a nanotube with initial radius $r_{0}$, we then define the dimensionless twist rate $\epsilon_{\circ}=\varphi r_{0} / l_{0}$ and use radian units for $\varphi$. Our results for the nanotube twisting energy $\Delta E_{t}$ are shown in Fig. 1(b). In the elastic regime, the torsional energy is well reproduced by

$$
\Delta E_{t} / l_{0}=\gamma \epsilon_{\circ}^{2}
$$

with $\gamma \approx 203.2 \mathrm{eV} / \AA$. The blue solid line in Fig. 1(b) for a circular cross-section follows Hook's law up to a large twist rate $\epsilon_{\circ, \max } \approx 0.26$. Comparing these results to those in Fig. 1(a), we note that a similar amount of deformation energy is associated with stretching and twisting of a nanotube. Also this idealistic prediction needs to be revisited.

Our calculations indicate that the circular nanotube cross-section is only metastable, since even a slight elliptical distortion induces flattening of the nanotube. Snap shots of the twist-induced nanotube flattening at different twist rates are shown in Fig. 1(c), and a hypothetical compact 3D arrangement of individually twisted nanotubes is depicted in Fig. 1(d). As seen in Fig. 1(b), the flattening is accompanied by a significant reduction of the total energy. On the other hand, flattened nanotubes are stable at twist rates far exceeding the elastic limit of the round nanotube, recovering in this way a significant portion of the maximum twist energy. Based on the maximum value of $\Delta E_{t} / l_{0}$ for a flattened nanotube in Fig. 1(b), we find that the deformation energy density in the system depicted in Fig. 1(d) may be as high as $J \approx 3 \mathrm{MJ} / \mathrm{kg}$.

When a finite bundle of straight nanotubes is twisted to a rope, each individual nanotube strand deforms to a coil of radius $\rho$, as shown in Fig. 2(a). As a nanotube rope is being twisted, each nanotube strand is subject to stretching, bending and twisting, and deforms accordingly. We calculated the deformation of individual nanotube strands in ropes containing 2 and 6 nanotubes using helical supercells. Our results for twist-induced shape changes in a double-helix (2-rope) and in a 6-rope are shown in Fig. 2(b). For those ropes, our results for the gravimetric deformation energy density $J$, based on DFTB, are presented in Fig. 2(c). These calculation have been constrained by the assumption that the interaction 


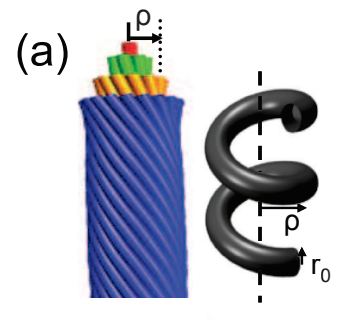

(b)
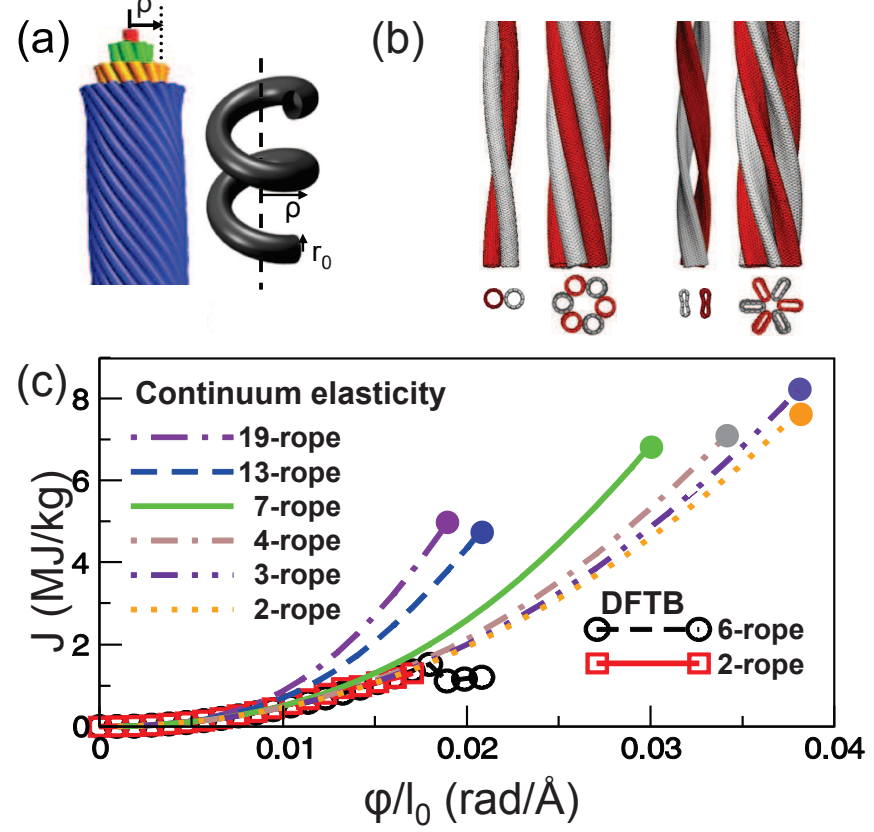

FIG. 2. (Color online) DFTB calculation results for deformation energy of twisted carbon nanotube ropes. (a) Schematic of a rope and the constituting nanotubes deformed to a coil. (b) Snap shots of structural changes, including the flattening of individual nanotubes, induced by twisting of a rope containing 2 and 6 nanotubes. (c) Gravimetric deformation energy density $J$ in twisted nanotube ropes as a function of the rope twist rate $\varphi / l_{0}$.

energy between neighboring nanotubes does not change as the rope is twisted, and by the imposed helical symmetry. In particular, we have also assumed that as a rope is twisted, the coil radius $\rho$ of each individual nanotube remains fixed at the initial value defined by the separation between the tube axis and the rope axis.

Also this assumption must be revisited, since it is intuitively clear that tightly twisting a rope may reduce its diameter. The equilibrium inter-tube distance, which is reflected in the rope diameter, is determined by the pairwise inter-tube repulsive interaction that balances the internal pressure inside a twisted rope. This pressure is caused by the resistance of each individual nanotube, which has been deformed to a coil, to twisting, stretching and bending. It is the microscopic counterpart to the well-known fact that excess water in a wet towel can be eliminated more efficiently by wringing than by squeezing. Since the internal pressure can be very high, the compression energy $\Delta E_{c}$ may reach a significant fraction of the deformation energy of a twisted nanotube rope in the elastic regime, prior to the onset of nanotube fusion.

To reliably estimate $\Delta E_{c}$ in a radially compressed rope, we replaced the rope by an infinite triangular lattice of $(18,0)$ carbon nanotubes with sixfold symmetry. We then used DFT calculations to optimize the nanotube geometry in a wide range of inter-tube distances $d$ far
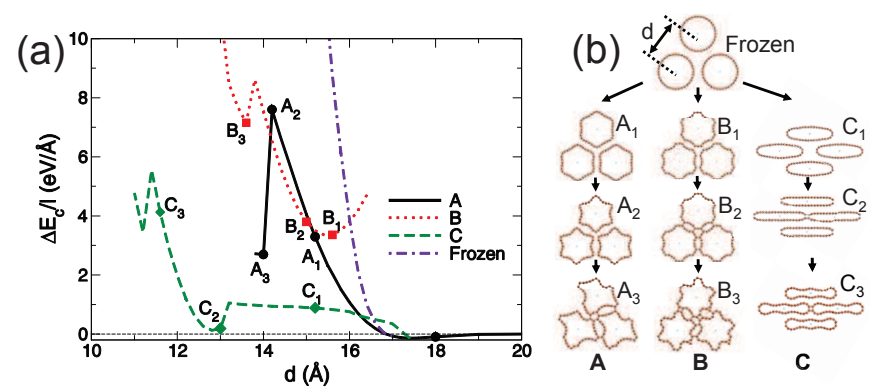

FIG. 3. (Color online) Structural changes and deformation energy of laterally compressed nanotube arrays, establishing the limits of elasticity and reversibility. (a) Compression energy per nanotube segment $\Delta E_{c} / l$ in an infinite nanotube lattice as a function of the inter-tube separation $d$. (b) Equilibrium geometries of stable nanotube array phases subject to different degrees of compression.

below the equilibrium value $d_{0}(18,0)=17.45 \AA$. Our results for the compression energy per nanotube segment are presented in Fig. 3(a) and the different shape deformation pathways are illustrated in Fig. 3(b). These results not only provide an estimate of the compressionrelated deformation energy capacity, but also help us to identify the limits of the elastic regime of deformations and to characterize the plastic deformations outside this regime.

Assuming that the compression energy $\Delta E_{c}$ may be decomposed into pairwise inter-tube interactions, we consider in the linear regime of small deformations the simplified expression

$$
\Delta E_{c} / l_{0}=\delta \epsilon_{\perp}^{2}
$$

for a pair of nanotubes subject to the strain $\epsilon_{\perp}=1-d / d_{0}$ normal to their axes. Our results in Fig. 3 indicate that the compressed nanotube lattice exhibits very complex structural changes and may deform along different pathways, which we label $A, B$ and $C$. We can infer from Fig. 3(a) that compression along any of these pathways is always energetically less costly than that of a lattice of nanotubes with their equilibrium geometry for $d>d_{0}$ artificially frozen in.

Maintaining the sixfold symmetry of the lattice along pathway $A$, the circular cross-section of each nanotube first changes to a hexagon $\left(A_{1}\right)$ and then a star with sixfold symmetry $\left(A_{2}\right)$ under increasing pressure. We note that the deformation energy associated with nanotube compression may reach the same order of magnitude as the deformation energy associated with stretching and twisting of nanotubes. When subject to the maximum normal strain $\epsilon_{\perp, \max } \approx 19 \%$ at $d=14.1 \AA$, corresponding to a pressure $\gtrsim 30 \mathrm{GPa}$, the structure collapses as neighboring tubes start connecting by covalent bonds.

Interestingly, structure $A_{1}$ may change under compression to a similar structure $B_{2}$, which does not spontaneously recover the initial nanotube structure with a cir- 
cular cross-section under zero pressure. Rather, it relaxes to a different structure $B_{1}$ with a star cross-section. Covalent inter-tube interaction along the $B$ pathway sets on at the critical normal strain $\epsilon_{\perp} \approx 21 \%$, similar to the $A$ pathway.

The energetically least costly compression pathway $C$ is initiated by first applying anisotropic pressure. With increasing pressure and decreasing inter-tube distance, the nanotube cross-section deforms to an ellipse $\left(C_{1}\right)$ and then a ribbon $\left(C_{2}\right)$ at similar critical normal strain values as found for structures $A$ and $B$. As seen in Fig. 3(a), compression thus far along pathway $C$ requires much less energy than along pathways $A$ and $B$. Whereas the compression energy in the elastic regime is reproduced well by Eq. (4) with $\delta(A)=92.6 \mathrm{eV} / \AA$ for branch $A$, a much smaller value $\delta(C) \approx 25.0 \mathrm{eV} / \AA$ may be used to estimate energy changes in branch $C$. The effective pressure normal to the tube axis does not exceed $3 \mathrm{GPa}$ and the minimum inter-wall distance maintains a nearly constant value $\approx 2.9-3.0 \AA$ in this regime. Only further compression to a fully collapsed ribbon $\left(C_{3}\right)$ in the inelastic regime comes at a somewhat higher energy cost. Under still higher pressures, collapsed nanotubes should spontaneously transform to graphene nanoribbons of uniform width, especially when exposed to hydrogen atmosphere[28].

In a rope containing a finite number of $N$ nanotube strands, the mechanical deformation energy contains a combination of stretching, twisting, bending and compression components. In the following, we consider a rope segment of initial axial length $l_{0}$, same as that of a straight unstrained constituent nanotube. We further consider the ideal case, where the structure of the clamped rope ends does not change and the rope is twisted as a whole. The energy change associated with twisting this macroscopic rope about its axis, a system too large for atomistic calculations, can be estimated using continuum elasticity expressions in Eqs. (1)-(4) for the individual energy terms. This treatment ignores the atomic structure of the individual nanotube strands, which are represented by flexible cylinders with equilibrium inter-tube distance $d_{0}$. Upon twisting, each cylinder $i$ is being deformed to a coil of radius $\rho_{i}$, as seen in Fig. 2(a). The total energy investment to twist a rope segment may then be expressed as

$\Delta E / l_{0}=\alpha \sum_{i=1}^{N} \epsilon_{\|, i}^{2}+\beta \sum_{i=1}^{N}\left(r_{0} / R_{i}\right)^{2}+N \gamma \epsilon_{\circ}^{2}+\delta \sum_{i<j} \epsilon_{\perp, i j}^{2}$,

where the summation in the fourth term extends only over nearest-neighbor nanotube pairs $i j$, and

$$
\epsilon_{\perp, i j}=1-d_{i j} / d_{0}
$$

Even though $l_{0}$ does not change as the rope is twisted, each individual nanotube strand is being subject to the

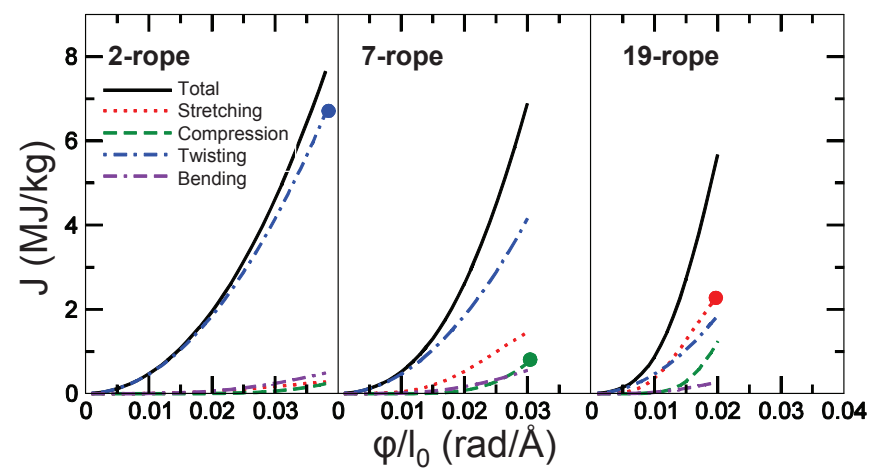

FIG. 4. (Color online) Contribution of individual terms of Eq. (5) towards the deformation energy density $J$ in nanotube ropes with 2, 7 and 19 strands. Solid dots terminate branches, where the elastic limit has been reached first.

axial strain

$$
\epsilon_{\|, i}=\sqrt{1+\left(\rho_{i} \varphi / l_{0}\right)^{2}}-1
$$

when each initially straight tube deforms to a coil within a rope that is subject to the twist rate $\varphi / l_{0}$. We note that the twist rate of each individual nanotube $\varphi / l_{0}$ is the same as that of the entire rope. Finally, the bending radius of each coil is given by [29] $R_{i}=\rho_{i}\left[1+\left(l_{0} /\left(\varphi \rho_{i}\right)\right)^{2}\right]$. Obviously, the deformation energy could be increased further if nanotubes within a rope could be twisted individually.

To estimate the deformation energy of a twisted nanotube rope, we optimize the inter-tube distances within the rope by minimizing the energy density $\Delta E / l_{0}$ in Eq. (5) for a given twist rate $\varphi / l_{0}$. The elastic response of the system results from the interplay between all components. For selected nanotube ropes, the total gravimetric deformation energy density $J$ as well as the individual energy components are shown in Fig. 4. Our energy decomposition provides not only an estimate of the maximum deformation energy density, but also allows us to identify the deformation channels that limit the elastic regime in each individual rope.

Our results for $J$ in ropes of $2 \leq N \leq 19$ nanotubes are presented in Fig. 2(c). For $(10,10)$ nanotubes, we used the equilibrium inter-tube distance $d_{0}=16.73 \AA$ and the value $\delta=25.0 \mathrm{eV} / \AA$ corresponding to the energetically least costly $C$ pathway in Fig. 3. The end points of the continuum elasticity results in Fig. 2(c) and Fig. 4 indicate the rope twist rate $\Delta E / l_{0}$, at which either the (experimental) stretching limit $\epsilon_{\|, \text {max }} \approx 12 \%$ or the twist limit $\epsilon_{\circ, \text { max }} \approx 0.26$ of individual tubes, or the compression limit $\epsilon_{\perp, \max } \approx 19 \%$ within the rope would be exceeded. Interestingly, the way the elastic limit is reached differs from rope to rope.

Our results in Fig. 4 show that the deformation energy at low twist rates is dominated by the twist energy, independent of the rope size. These results also show that 
the thinnest ropes with $N \leq 4$ nanotubes should fracture by torsion first. We find that stretching and compression contributions gain significance in wider ropes with $4 \lesssim N \lesssim 19$, where the elastic regime becomes limited by compression strain. This is intuitively clear from the everyday experience that wringing is more effective when the wet object is long and narrow. As seen in Fig. 4, in very wide ropes with $N \gtrsim 19$ nanotubes, the contribution of stretching grows significantly and becomes comparable to that of compression and twisting. In these wide ropes, according to Fig. 4, the nanotubes in the outermost rope layer break by stretching first, well before other decay mechanisms including compression would occur. In all ropes considered here, we found the bending deformation energy to be negligible.

In conclusion, we determined the deformation mechanism and deformation energy density in arrays of twisted carbon nanotubes and nanotube ropes which constitute a torsional spring. Using $a b$ initio and parameterized density functional calculations, we identified structural changes in these systems and determined their elastic limits. We found that the deformations of twisted nanotube ropes are determined by an interplay between twisting, stretching, bending and compression of individual nanotubes. We quantified the corresponding energy contributions and showed that their relative role changes with the number of nanotubes in the rope.

We acknowledge valuable contributions to the computational approach by Dong-Bo Zhang and Traian Dumitrica. This work was funded by the National Science Foundation Cooperative Agreement \#EEC-0832785, titled "NSEC: Center for High-rate Nanomanufacturing". GS was partly supported by the European Centre for Emerging Materials and Processes Dresden (ECEMP, project number: 10 13857/2379). The first author's visit to MSU was partially funded by the DAAD. Computational resources for this project were provided by the ZIH Dresden and the Michigan State University HighPerformance Computer Center. We thank T. Moore for assistance with the visualization of structures.

* E-mail: tomanek@pa.msu.edu

[1] A. Jorio, M. Dresselhaus, and G. Dresselhaus, eds., Carbon Nanotubes: Advanced Topics in the Synthesis, Structure, Properties and Applications, Topics in Applied Physics No. 111 (Springer, Berlin, 2008).

[2] M.-F. Yu, O. Lourie, M. J. Dyer, K. Moloni, T. F. Kelly, and R. S. Ruoff, Science 287, 637 (2000).
[3] M.-F. Yu, B. S. Files, S. Arepalli, and R. S. Ruoff, Phys. Rev. Lett. 84, 5552 (2000).

[4] D. A. Walters, L. M. Ericson, M. J. Casavant, J. Liu, D. T. Colbert, K. A. Smith, and R. E. Smalley, Appl. Phys. Lett. 74, 3803 (1999).

[5] F. A. Hill, T. F. Havel, and C. Livermore, J. Micromech. Microeng. 19, 094015 (2009).

[6] D. Li, W. F. Paxton, R. H. Baughman, T. J. Huang, J. F. Stoddart, and P. S. Weiss, MRS Bull. 34, 671 (2009).

[7] J. Foroughi, G. Spinks, G. Wallace, J. Oh, M. Kozlov, S. Fand, T. Mirfakhrai, J. Madden, M. K. Shin, S. J. Kim, and R. Baughman, Science 334, 494 (2011).

[8] G. Overney, W. Zhong, and D. Tomanek, Z. Phys D: Atoms, Molecules and Clusters 27, 93 (1993).

[9] B. I. Yakobson, C. J. Brabec, and J. Bernholc, Phys. Rev. Lett. 76, 2511 (1996).

[10] B. I. Yakobson, Appl. Phys. Lett. 72, 918 (1998).

[11] B.-W. Jeong, J.-K. Lim, and S. B. Sinnott, Appl. Phys. Lett. 90, 023102 (2007).

[12] B.-W. Jeong, J.-K. Lim, and S. B. Sinnott, J. Appl. Phys. 101, 084309 (2007).

[13] D. Qian, W. K. Liu, and R. S. Ruoff, Comp. Sci. Technol. 63, 1561 (2003).

[14] J. Alford, B. Landis, and J. Mintmire, Int. J. Quantum Chem. 105, 767 (2005).

[15] F. A. Hill, T. F. Havel, and C. Livermore, Nanotechn. 20, 255704 (2009).

[16] C. Q. Ru, Phys. Rev. B 62, 10405 (2000).

[17] J. M. Soler, E. Artacho, J. D. Gale, A. García, J. Junquera, P. Ordejón, and D. Sánchez-Portal, J. Phys: Cond. Mat. 14, 2745 (2002).

[18] D. M. Ceperley and B. J. Alder, Phys. Rev. Lett. 45, 566 (1980).

[19] J. P. Perdew and A. Zunger, Phys. Rev. B 23, 5048 (1981).

[20] N. Troullier and J. L. Martins, Phys. Rev. B 43, 1993 (1991).

[21] E. Artacho, D. Sánchez-Portal, P. Ordejón, A. García, and J. M. Soler, Phys. Stat. Sol. (b) 215, 809 (1999).

[22] D. Porezag, T. Frauenheim, T. Köhler, G. Seifert, and R. Kaschner, Phys. Rev. B 51, 12947 (1995).

[23] D.-B. Zhang, M. Hua, and T. Dumitrica, J. Chem. Phys. 128, 084104 (2008).

[24] D.-B. Zhang and T. Dumitrica, Appl. Phys. Lett. 93, 031919 (2008).

[25] D.-B. Zhang, R. D. James, and T. Dumitrica, Phys. Rev. B 80, 115418 (2009).

[26] L. Zhechkov, T. Heine, S. Patchkovskii, G. Seifert, and H. A. Duarte, J. Chem. Theory and Computation 1, 841 (2005).

[27] E. Ertekin and D. C. Chrzan, Phys. Rev. B 72, 045425 (2005).

[28] S. Berber and D. Tománek, Phys. Rev. B 80, 075427 (2009).

[29] D. Teich, G. Seifert, S. Iijima, and D. Tománek, Phys. Rev. Lett. 108, 235501 (2012). 